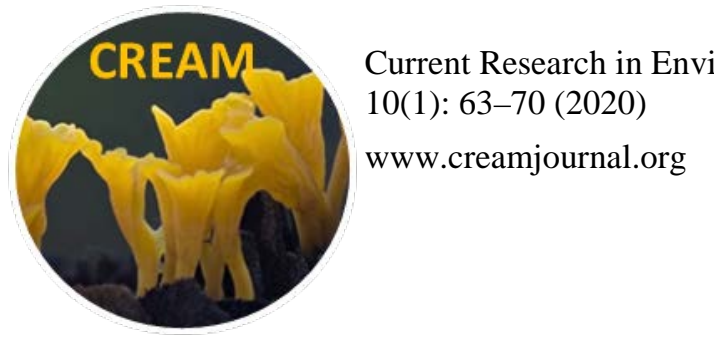

\author{
Article \\ Doi 10.5943/cream/10/1/7
}

\title{
How are basidiospore size measurements affected by drying?
}

\section{Dramani $\mathbf{R}^{*}$, Hegbe ADMT ${ }^{1}$, Tabe $\mathbf{A}^{1}$, Badou AS ${ }^{1}$, Furneaux BR $^{2}$, Ryberg $\mathbf{M}^{2}$ and Yorou NS ${ }^{1}$}

${ }^{1}$ Tropical Mycology and Plant-Soil Fungi Interactions, Laboratory of Ecology, Botany and plant Biology, Faculty of Agronomy, University of Parakou, 03 BOX: 125, Parakou, Benin

${ }^{2}$ Systematic Biology program, Department of Organismal Biology, Uppsala University, Norbyvägen 17D, 75236

Uppsala, Sweden

Dramani R, Hegbe ADMT, Tabe A, Badou AS, Furneaux B, Ryberg M, Yorou NS 2020 - How are basidiospore size measurements affected by drying? Current Research in Environmental \& Applied Mycology (Journal of Fungal Biology) 10(1), 63-70, Doi 10.5943/cream/10/1/7

\begin{abstract}
Spore size plays a crucial role in the demarcation of fungal species. Spore size is measured from either fresh or, more frequently, dried fungal specimens. In the present study, we investigate the impact of drying on the basidiospore size of three ectomycorrhizal species, and the bias it can generate on the taxonomic identification of fungal species. We measured a total of 1109 basidiospores of Amanita masasiensis, 2322 basidiospores of Lactifluus gymnocarpoides, and 920 basidiospores of Russula congoana from fresh sporocarps and spore prints. After specimens and spore prints were dehydrated for $24 \mathrm{~h}$ at $50^{\circ} \mathrm{C}$, we measured 1200 basidiospores of $A$. masasiensis, 2348 basidiospores of $L$. gymnocarpoides and 1150 basidiospores of $R$. congoana. The effects of drying on length, width, and length/width quotient (Q) values were tested using a linear mixed model to control for inter-individual variation. Drying reduced the basidiospore length and width of A. masasiensis by $10 \%$ and $16 \%$ respectively, resulting in a $7 \%$ increase in the quotient Q, while both the length and width of basidiospores of $L$. gymnocarpoides and $R$. congoana were reduced by $3 \%$ and $6 \%$ respectively, with no change in Q. This study suggests that greater attention should be given to the condition of the spores when spore size is used as a taxonomically discriminative characteristic. This is especially relevant for the taxonomy of cryptic species, where other discriminative characteristics may be lacking.
\end{abstract}

Key words - basidiospores size - dehydration - fungi - taxonomy

\section{Introduction}

Spores are reproductive propagules whose color, shape, and size play a crucial role in taxon delimitation in the traditional taxonomy of fungi. The first classification of fungi was based on the use of spore color to define major taxonomic groups (Fries 1821), and up until the molecular boom in fungal taxonomy (White et al. 1990, Ryberg et al. 2009, Seifert 2009, Abarenkov et al. 2010, Nilsson 2011). Spore size has probably been the most frequently used features in the traditional taxonomy of fungi (Togashi \& Oda 1934, Romagnesi 1961, Bas 1969, EinheIlinger 1985, Parmasto \& Parmasto 1987, 1992, Parmasto 1989). Though molecular techniques are powerful tools for detecting cryptic, uncultivable and microscopic mycodiversity (Begerow et al. 2010), spore features still remain crucial for species discrimination, and shape the morphological species concept even 
within the context of modern mycology (Hibbett et al. 2011). Although spore size and shape may be characteristic for a species, they may also vary based on factors such as the age and size of the sporocarp, micro-climatic conditions, and the harvest period (Togashi \& Oda 1934, Parker-Rhodes 1949).

To measure spore size, spore samples can be taken from fresh or dried specimens, or even from spore prints (spores that have been released from the sporocarp; (Parmasto \& Parmasto 1987). Materials are often dried for long term preservation and conservation of voucher specimens (Agerer et al. 2000), and because of constraints such as availability of microscopic equipment at or close to collecting sites and time constraints in connection to collecting material, it can be difficult to investigate a large number of spore samples and specimens in the fresh condition. When reexamining vouchers, dried materials are almost always used. Most, if not all, traditional taxonomical works on fungi are performed using dried materials through refreshment of fungal cells by mean of chemicals (Clémençon 1997). Intensive drying may alter and collapse fungal spores, thus making species identification difficult (Yoshito et al. 2004), whilst the loss of water during the drying process may be correlated with the thickness of the spore membrane. Drying methods and conditions may change the shape and dimensions of dried spores compared to fresh ones. As such, it is unlikely that the range limits of spore size used to taxonomically characterize a species are identical in fresh and dried conditions.

We here investigate whether there is a shift in spore size and shape from fresh to dried spores, in order to further morphological species recognition. Understanding the impact of drying on species delimitation is crucial, especially for cryptic taxa that do not display a clear hiatus in spore dimensions.

\section{Materials \& Methods}

We investigated three taxonomically well circumscribed and easy-to-identify fungal species in two different orders in the Agaricomycotina: Lactifluus gymnocarpoides (Verbeken) Verbeken, Russula congoana Pat. (Russulaceae; Russulales), and Amanita masasiensis Härk. \& Saarim (Amanitaceae; Agaricales). Specimens of the three taxa (Fig. 1) were collected in GuineoSoudanian ectomycorrhizal-dominated woodlands (Yorou et al. 2014) in Benin from June to September 2016. Color pictures of the specimens were taken using a digital camera (Canon 70EOD). Preliminary morphological data were recorded and identification was confirmed on fresh specimens following standard description methods for the target genera: Verbeken \& Walleyn (2010) for Lactifluus, Buyck (1993, 1994) for Russula and Härkhönen et al. (2003) and Tulloss \& Yang (2017) for Amanita. Spore prints (Clémençon 2009) were collected from representative sporocarp by placing the pileus hymenium-down on a light-colored piece of paper for 12-18 hours. Specimens and spore prints were dried using an electric dryer (Dorrex Stockli 0076) at a temperature of $50^{\circ} \mathrm{C}$ for 24 hours (De Kesel et al. 2002) to prevent damage on fungal structures and be sure for complete drying of specimens. After complete dehydration of the specimens, the sporocarp and spore prints were packed in hermetically sealed minigrip-type polyethylene bags with their respective labels to prevent re-moistening. All labeled dried specimens are deposited at the mycological herbarium of the University of Parakou (UNIPAR, Thiers 2019).

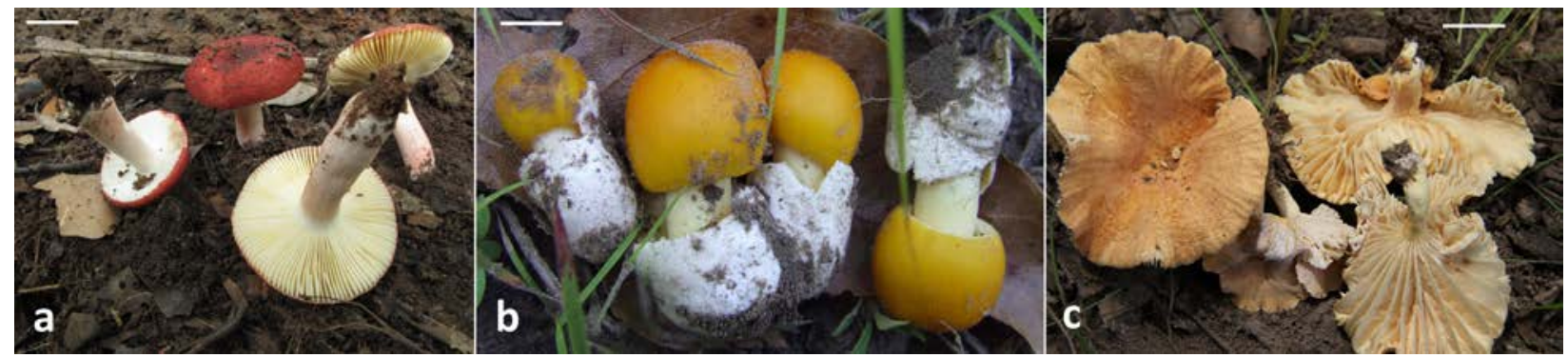

Fig. 1 - Color images of the species investigated. a Russula congoana. b Amanita masasiensis. c Lactifluus gymnocarpoides. Scale Bars $=1 \mathrm{~cm}$. 


\section{Microscopic examination of specimens}

Basidiospores from fresh spore prints and from fresh sporocarps were examined microscopically, and then the same specimens and their spore prints were examined again after drying. For sporocarp, fine pieces of lamellae were excised with a razor blade under a Leica M6 stereomicroscope. Excised lamellae, or a sample of basidiospores from a spore print, were mounted in tap water (for fresh material) or in $3 \% \mathrm{KOH}$ in order to replenish the tissues (in case of dried specimens; (Clémençon 2009). The preparation was then observed by mean of a Leica DM270M light microscope equipped with a drawing tube and a scaled ocular. Line drawings and measurements of the basidiospores were performed at 1000× magnification under normal light. Members of Russulaceae generally have ornamented (warted, spiny or winged) basidiospores (Verbeken \& Walleyn 2010). For both L. gymnocarpoides and R. congoana, measurements did not include the ornamentations. Basidiospore measurements were recorded to the nearest $\mu \mathrm{m}$.

\section{Spore size expression and statistics}

The length $(\ell)$ and the width (w) of each selected basidiospore were measured (Fig. 2), where $\ell$ is the size along the longest axis, and $w$ is the size along an axis perpendicular to $\ell$. Basidiospore size ranges are expressed as $\left(\ell_{\text {ext.min }}\right) \ell_{\min }-\ell_{\max }\left(\ell_{\text {ext.max }}\right) \times\left(\mathrm{W}_{\text {ext.min }}\right) \mathrm{W}_{\min }-\mathrm{W}_{\max }\left(\mathrm{W}_{\text {ext.max }}\right)$, and the average size as $\ell_{M} \times w_{M}$, where "ext.min" denotes the extreme minimum, "min" denotes the $5^{\text {th }}$ percentile value, "max" denotes the $95^{\text {th }}$ percentile value, "ext.max" denotes the extreme maximum, and " $\mathrm{M}$ " denotes the mean. The quotient $\mathrm{Q}=\ell / \mathrm{w}$ describes the shape of the basidiospore, and is likewise expressed as the range $\left(\mathrm{Q}_{\text {ext.min }}\right) \mathrm{Q}_{\min }-\mathrm{Q}_{\max }\left(\mathrm{Q}_{\text {ext.max }}\right)$ and mean value $\mathrm{Q}_{\mathrm{M}} \mathrm{N}$ and $\mathrm{SD}$ represent the number of basidiospores sampled per specimen and the standard deviation of the number of basidiospores per specimen, respectively.

Length, width, and Q values were log-transformed, and then fit to a linear mixed model using the "nlme" package (Pinheiro et al. 2017) in R version 3.3.2 (R Core Team 2017). The state (fresh vs. dry) and species, as well as the interaction between them, were modeled as fixed effects, and the specimen the spores came from was modeled as a random effect. Statistical tests were performed using the "multcomp" package (Hothorn et al. 2008) to evaluate the effects of drying on basidiospore size and shape for each species, and to test for differences in effects between species. Changes in the size and shape distribution of basidiospores before and after drying were visualized using the "beanplot" package (Kampstra 2008).
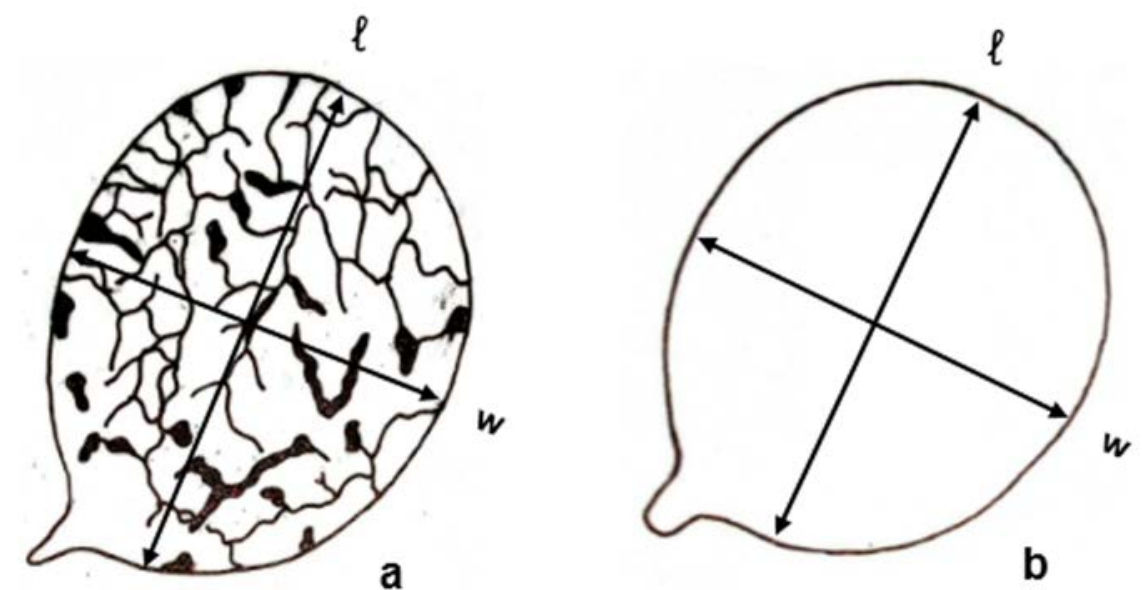

$5 \mu \mathrm{m}$

Fig. 2 - Measuring basidiospores dimensions. a basidiospore of L. gymnocarpoides. b basidiospore of A. masasiensis. 


\section{Results}

\section{Sampling efforts}

We investigated 24 specimens of A. masasiensis, 47 of L. gymnocarpoides and 23 specimens of $R$. congoana, for a total of 94 specimens investigated. The difference in number of investigated specimens between species depended upon the availability of specimens during the sampling period. We attempted to investigate as many specimens as possible.

\section{Descriptive statistics and distribution of basidiospore sizes in the investigated species}

Due to time constraints when working with fresh material we investigated more spores from dried material than from fresh material. Spore size metrics for all three targeted species are summarized in Table 1 below. The size and shape distribution of spores for the different species are shown in Fig. 3.

\section{Impact of drying on basidiospore size of targeted species}

In general, drying reduced the size of the spores in all three species (Fig. 3). After drying, the length and width of $A$. masasiensis basidiospores decreased by an average of $10 \%$ ( $p<0.0001$, 95\% confidence interval of mean (CIOM) 9\%-11\%) and 16\% (p < 0.0001, CIOM 15\%-17\%). There was a 3\% mean reduction in the length and width of the basidiospores of L. gymnocarpoides $(\ell, \mathrm{p}<0.0001$, CIOM $2 \%-4 \%$; w, p $<0.0001$, CIOM 3\%-4\%). In $R$. congoana, the length and width of basidiospores were reduced by $6 \%(\ell, \mathrm{p}<0.0001$, CIOM 5\%-7\%; w, p $<0.0001$, CIOM 5\%-7\%) after drying.

Table 1 Basidiospore size metrics for the target species. Q = quotient length/width expression of basidiospore; $\mathrm{N}=$ number of basidiospores sampled per specimen; $\mathrm{SD}=$ standard deviation of the number of basidiospores per specimen.

\begin{tabular}{llll}
\hline & A. masasiensis & L. gymnocarpoides & R. congoana \\
\hline Specimens & 24 & 47 & 23 \\
Total fresh basidiospores & 1109 & 2322 & 920 \\
Total dry basidiospores & 1200 & 2348 & 1150 \\
Basidiospore size, fresh & $(6) 7-13(15) \times$ & $(6) 7-9(11) \times$ & $(6) 7-10(11) \times$ \\
$(\mu \mathrm{m})$ & $(5) 6-9(10)$ & $(5) 6-8(9)$ & $(5) 6-8(9)$ \\
Mean size, fresh $(\mu \mathrm{m})$ & $10.1 \times 7.1$ & $8.0 \times 7.0$ & $8.3 \times 7.0$ \\
Q, fresh & $(0.71) 1.43-1.75(4.50)$ & $(1.00) 1.14-1.33(1.80)$ & $(1.00) 1.14-1.33(1.57)$ \\
Mean Q, fresh & 1.44 & 1.16 & 1.20 \\
N, fresh & $7-50$ & $39-50$ & 40 \\
SD, fresh & 10.9 & 2.3 & 0.0 \\
Basidiospore size, dry & $(5) 7-11(12) \times$ & $(6) 7-9(10) \times$ & $(6) 7-9(10) \times$ \\
$(\mu m)$ & $(4) 5-8(9)$ & $(5) 6-7(9)$ & $(5) 6-8(9)$ \\
Mean size, dry $(\mu \mathrm{m})$ & $9.0 \times 5.9$ & $7.8 \times 6.7$ & $7.8 \times 6.5$ \\
Q, dry & $(0.60) 1.57-2.00(2.40)$ & $(0.88) 1.14-1.33(1.60)$ & $(0.86) 1.17-1.33(1.67)$ \\
Mean Q, dry & 1.54 & 1.16 & 1.20 \\
N, dry & 50 & $48-50$ & 50 \\
SD, dry & 0.0 & 0.3 & 0.0 \\
\hline
\end{tabular}

\section{The Q Ratio (Length/Width)}

Drying increased the $\mathrm{Q}$ ratio $(\ell / \mathrm{w})$ of the basidiospores of $A$. masasiensis by $7 \%(\mathrm{p}<0.0003$, CIOM 6\%-9\%), but had no significant effect on basidiospores shape of L. gymnocarpoides ( $\mathrm{p}=$ 0.3 , CIOM 0\%-1\%) and $R$. congoana (p = 0.85, CIOM -1\%-2\%). 


\section{Interspecific assessment of the impact of drying on basidiospores}

The interaction of species with drying was found to be significant for all three measure parameters $(\ell, \mathrm{p}<0.0001 ; \mathrm{w}, \mathrm{p}<0.0001$; and $\mathrm{Q}, \mathrm{p}<0.001)$. Post-hoc tests found that all three species differed in the effect of drying on length and width, with $A$. masasiensis showing the greatest effects, and L. gymnocarpoides showing the smallest. There was no statistically significant difference between L. gymnocarpoides and R. congoana in the effect of drying on Q (p $=0.99$ ). This is not surprising, since neither of these species showed any consistent change in $Q$ after drying.
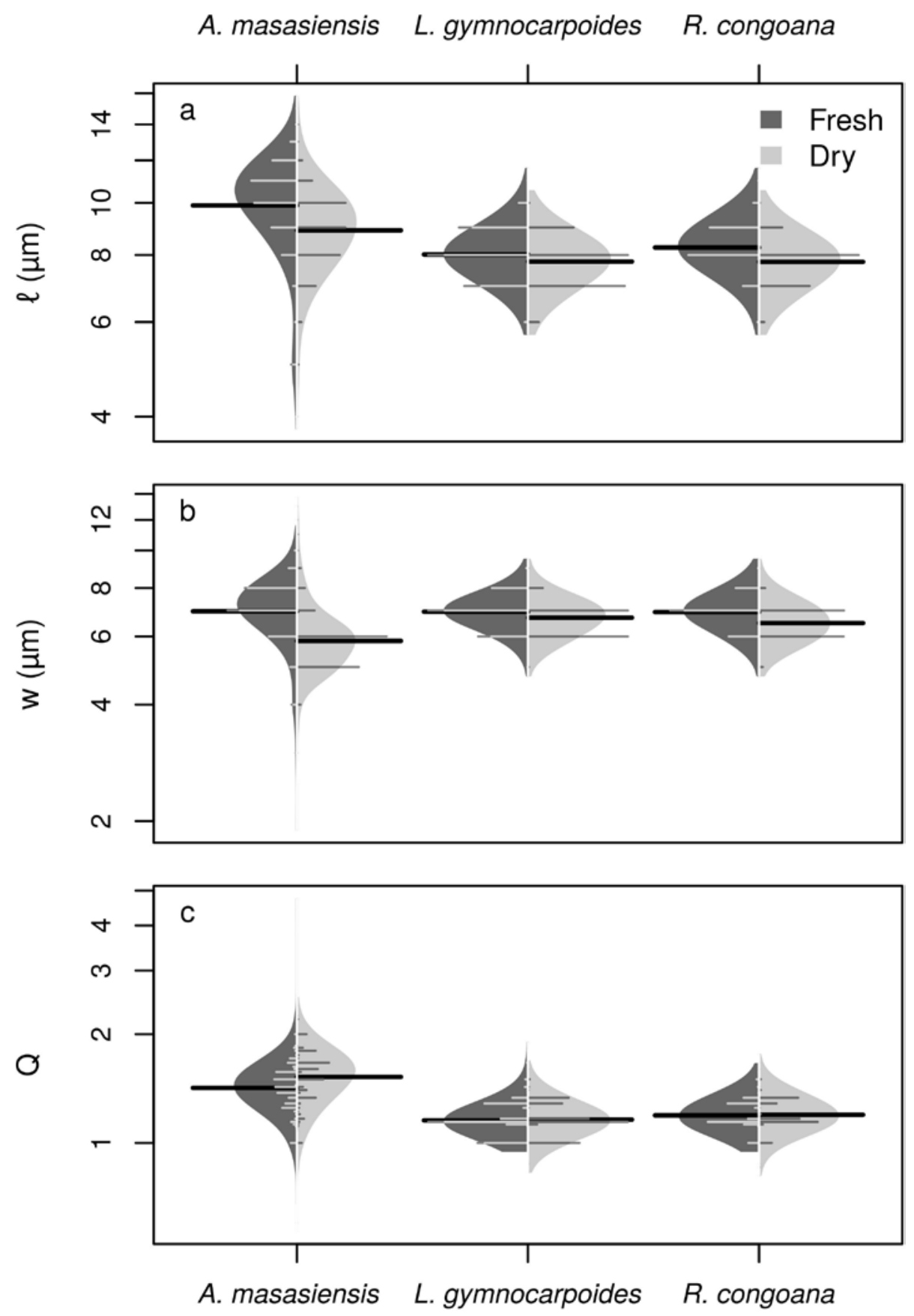

Fig. 3 - Beanplots of basidiospores length (a), width (b) and Q ratio (length/width; c) distributions of targeted species. In all graphs, a shaded kernel density plot (Gaussian bandwidth $=0.1 \log$ units) of the distribution before drying is shown in dark gray on the left, and after drying in light gray on the right. Vertical axes are log-transformed. Thin lines within the distributions show the observed values. Thick black lines show the means of the distributions. 


\section{Discussion}

This study reveals that drying affects the basidiospore sizes, suggesting that great care should be taken during species demarcation, especially for cryptic species. For genera such as Amanita that include toxic taxa, misidentification of species may lead to irreversible intoxication (Härkhönen et al. 1995), although edible and fatally toxic species are typically differentiated by more than spore characteristics. Original descriptions of species have been performed using dried specimens by mycologists who are not in a position to obtain fresh material. As notes on detailed morphological features (color, smell, taste) are often lacking, and in the absence of facilities to perform molecular investigations, spore dimensions may play a critical role in the description, and discrepancies in basidiospore measures between fresh and dried material may lead to taxonomical confusions. The dimensions of dried basidiospore reported here for A. masasiensis match well with the original descriptions of this species (Härkhönen et al. 1994, Tulloss \& Yang 2017). However, basidiospore sizes of fresh material of $A$. masasiensis are similar to those of $A$. tanzanica (Tulloss \& Yang 2017). Both close species may be misidentified if the taxonomy is solely based on dried basidiospores.

There have been very few studies that address drying as a factor on basidiospore size. (Clémençon 1979) reported the rapid decline of spore size during basidiomata desiccation, a phenomenon that is also demonstrated in the present studies (Fig. 3). Other environmental factors that control the intra-specific variation of spore size include the humidity, temperature, and light conditions of the habitat, and the substrate on which the species grows (Clémençon 1979, Kauserud et al. 2008). Such differences are here accounted for by making a pairwise comparison using the same sporocarps for both the fresh and dried treatment (Table 1).

Although drying reduced basidiospore size in all species, there was no impact on the $\mathrm{Q}$ value of basidiospores for L. gymnocarpoides and R. congoana (Russulaceae), but for A. masasiensis the $\mathrm{Q}$ value was also impacted. So, considering the standards defined by Neville \& Poumarat (2004) to characterize the shape of basidiospore based on their Q value in "Amaniteae", it's obvious that this characterization is biased if measurements were done on dried spores. The length and width reduction in the same proportions respectively 3\% and 6\% for L. gymnocarpoides and $R$. congoana justifies the non-variation observed in their $\mathrm{Q}$ value, unlike A. masasiensis.

Among other factors that explain the change in basidiospore size and shape between investigated species, we speculate that the structure and content of the basidiospore cell walls and possibly the basidiospore ornamentation are key factors controlling water loss during drying process. Lactifluus gymnocarpoides and $R$. congoana, along with other members of the Russulaceae, have strongly ornamented (warted, spiny or winged) basidiospores (Buyck 1993, Verbeken \& Buyck 2001). In addition, the basidiospore ornamentation in Russulaceae is starchy, or amyloid (Verbeken \& Walleyn 2010), which is evidenced by a blue to dark blue colour in Melzer's reagent. Both L. gymnocarpoides and R. congoana have warted basidiospores (Buyck 1994, Verbeken \& Buyck 2001) while A. masasiensis has smooth and inamyloid basidiospores (Härkhönen et al. 1994). More likely the basidiospore ornamentation in L. gymnocarpoides and $R$. congoana may have played a buffer role and reduce the loss of water during drying process. The thickness of the cell wall may also play a role, but this was not measured in the current study.

Our results caution against comparing spore measures based on fresh and dried material, and highlight the need to clearly indicate under which condition the spores were measured when describing taxa. Moreover, according to Raja et al. (2017), for a complete delimitation of taxa, it would be better to associate the micromorphological characteristics to those molecular focused on the use of DNA barcoding technique, revolutionary of the taxonomy.

\section{Acknowledgments}

Fieldwork, specimen sampling and a large part of laboratory work were made possible by financial support from FORMAS (Sweden) grant $n^{\circ}$ 226-2014-1109. Microscopic investigations were possible by mean of laboratory equipment granted to the University of Parakou (Benin) by the 
Volkswagen Foundation (Grant no. 90-127). We are also very grateful to the anonymous reviewers for their valuable comments in improving the early manuscript.

\section{References}

Abarenkov K, Henrik Nilsson R, Larsson KH, Alexander IJ et al. 2010 - The Unite database for molecular identification of fungi-recent updates and future perspectives. New Phytologist 186, 281-285.

Agerer R, Ammirati J, Blanz P. 2000 - Always deposit a voucher. Mycological research 104, 642644.

Bas C. 1969 - Morphology and subdivision of Amanita and a monograph of its section Lepidella. Persoonia 5, 285-579.

Begerow D, Nilsson H, Unterseher M, Maier W. 2010 - Current state and perspectives of fungal DNA barcoding and rapid identification procedures. Applied Microbiology and Biotechnology 87, 99-108.

Buyck B. 1993 - Russula I (Russulaceae). In: Rammeloo J, Heinemann P (Ed) Flore illustrée des Champignons d'Afrique Centrale, 335-408.

Buyck B. 1994 - Russula II (Russulaceae). In : Rammeloo J, Heinemann P (Ed) Flore illustrée des Champignons d'Afrique Centrale, 411-542.

Clémençon H. 1979 - Biometrische untersuchungen zur variabiliât der basidiosporen. Sydowia Beihefte 8, 110-138.

Clémençon H. 1997 - Anatomie der Hymenomyceten. Lausanne.

Clémençon H. 2009 - Methods for working with macrofungi: laboratory cultivation and preparation of larger fungi for light microscopy. IHW VErlag.

De Kesel A, Codjia JTC, Yorou NS. 2002 - Guide des champignons comestibles du Benin. ISBN 99919-953-2-3

Einhellinger A. 1985 - Die Gattung Russula in Bayern. - Hoppea. Denkschriften der Regensburgischen Botanischen Gesellschaft 43, 5-286.

Fries EM. 1821 - Systema mycologicum. Lundae 1, 1-520.

Härkhönen M, Niemelä T, Mwasumbi L. 2003 - Tanzanian mushrooms. Edible, harmful and other fungi. Helsinki: Finish Museum of Natural History, Norrlinia.

Härkhönen M, Saarimaki T, Mwasumbi L. 1994 - Tanzania mushrooms and their uses. Some reddish edible and poisonous Amanita species. Karstenia 34, 47-60.

Härkhönen M, Saarimäki T, Mwasumbi L. 1995 - Edible mushrooms of Tanzania. Karstenia 35, 191.

Hibbett DS, Ohman A, Glotzer D, Nuhn M et al. 2011 - Progress in molecular and morphological taxon discovery in Fungi and options for formal classification of environmental sequences. Fung Biology Reviews 25, 38-47.

Hothorn T, Bretz F, Westfall P. 2008 - Multcomp: Simultaneous Inference in General Parametric Models. Biometrical Journal 50, 346-363.

Kampstra P. 2008 - Beanplot: A Boxplot Alternative for Visual Comparison of Distributions. Journal of Statistical Software, Code Snippets 28, 1-9.

Kauserud H, Colman JE, Ryvarden L. 2008 - Relationship between basidiospore size, shape and life history characteristics: a comparision of polypores. Fungal Ecology 1, 19-23.

Neville P, Poumarat S. 2004 - Amaniteae. Amanita, Limacella \& Torrendia. Fungi Europaei ISBN 88-901057-3-9, 1120p.

Nilsson J. 2011 - Proteome-wide evidence for enhanced positive Darwinian selection within intrinsically disordered regions in proteins. Genome Biology 12, R65.

Parker-Rhodes AF. 1949 - The basidiomycetes of Skokholm Island. II. Genetical implications of spores' measurements in two Agarics. New Phytologist 48, 382-389.

Parmasto E. 1989 - The size of the basidiospres in the families of Agaricales., Tenth Congress of European Mycologists. Abstracts. Tallinn. 
Parmasto E, Parmasto I. 1987 - Variation of basidiospores in the hymenomycetes and its significance to their taxonomy. Berlin: Bibliotheca mycologica.

Parmasto E, Parmasto I. 1992 - Size and shape of basidiospores in the hymenomycetes. Mycologia Helvetica 5, 47-78.

Pinheiro J, Bates D, DebRoy S, Sarkar D, Team RC. 2017 - nlme: Linear and Nonlinear Mixed Effects Models. $\mathrm{R}$ package version 3, 1-131. URL: https://CRAN.Rproject.org/package $=$ nlme

Raja HA, Miller AN, Pearce CJ, Oberlies NH. 2017 - Fungal Identification Using Molecular Tools: A Primer for the Natural Products Research Community. Journal of Natural Products 80, $756-770$.

R Core Team. 2017 - R: A Language and Environment for Statistical Computing. R Foundation for Statistical Computing, Vienna, Austria.

Romagnesi H. 1961 - Biométrie, écologie et spécification. Bulletin de la Société Mycologique Française 77, 325-330.

Ryberg M, Kristiansson E, Sjokvist E, Nilsson RH. 2009 - An outlook on the fungal internal transcribed spacer sequences in GenBank and the introduction of a web-based tool for the exploration of fungal diversity. New Phytologist 181, 471-477.

Seifert KA. 2009 - Progress towards DNA barcoding of fungi. Molecular Ecology Resources 9, 83-89.

Thiers B. 2019 - (continuously updated). Index Herbariorum: A global directory of public herbaria and associated staff. New York Botanical Garden's Virtual Herbarium. http://sweetgum.nybg.org/science/ih/

Togashi K, Oda K. 1934 - Spores-sizes variability in subsequent spore prints of some hymenomycetous fungi. Transaction of the sapporo natural hystory society 13, 121-125.

Tulloss RE, Yang ZL. 2017 - US- FL, GA, AL. In: Tulloss RE, Yang ZL (Eds), Amanitaceae studies.

Verbeken A, Buyck B. 2001 - Biodiversity and ecology of tropical ectomycorrhizal fungi in Africa. In: Watling R, Frankland JC, Ainsworth AM, Isaac S, Robinson R (Ed) Tropical Mycology, Macromycetes CAB International, Wallingfort, UK, 11-24.

Verbeken A, Walleyn R. 2010 - Monograph of Lactarius in Tropical Africa. National Botanic Garden of Belgium Fungus Flora of Tropical Africa.

White TJ, Bruns T, Lee S, Taylor J. 1990 - Amplification and direct sequencing on fungal ribosomal RNA genes for phylogenetics. In: Innis MA GD, Sninsky JJ, White TJ (Ed) PCR Protocols: A Guide to Methods and Application, Academic Press, New York, 315-322.

Yorou NS, Koné NA, Guissou KML, Guelly AK et al. 2014 - Biodiversity and sustainable use of wild edible fungi in the Sudanian centre of endemism: a plea for valorization. In: Ectomycorrhizal symbiosis in tropical and Neotropical Forests. Taylor and Francis Group BR (Ed) Bâ, AM. Florida, 241-269.

Yoshito S, Manami K, Susumu T. 2004 - Molecular phylogeny of Russulaceae (Basidiomycetes; Russulales) inferred from the nucleotide sequences of nuclear large subunit rDNA. Mycoscience 45, 303-316. 